\title{
Modulation of pro-inflammatory and pro-resolution mediators by $\gamma$-linolenic acid: an important element in radioprotection against ionizing radiation
}

\author{
Rangachar Poorani ${ }^{1,2}$, Anant N. Bhatt ${ }^{1}$, Undurti N. Das 2,3
}

${ }^{1}$ Institute of Nuclear Medicine and Allied Sciences, DRDO, Delhi, India

${ }^{2}$ BioScience Research Centre and Department of Medicine, GVP Hospital and Medical

College, Visakhapatnam, India

${ }^{3}$ UND Life Sciences, Battle Ground, WA, USA

Submitted: 15 November 2018

Accepted: 16 January 2019

\author{
Corresponding author: \\ Undurti N. Das \\ UND Life Sciences \\ 2221 NW $5^{\text {th }}$ St. \\ Battle Ground \\ WA 98604, USA \\ E-mail: Undurti@bsrc.co.in
}

Arch Med Sci 2020; 16 (6): 1448-1456

DOI: https://doi.org/10.5114/aoms.2020.92469

Copyright (c) 2020 Termedia \& Banach

\section{Abstract}

Introduction: The current study explored the radio-protective property of $\gamma$-linolenic acid (GLA) in C57BL/6J mice against low linear energy transfer ionizing radiation (IR; $\mathrm{X}$-rays) and its modulatory effect on the production of lipid mediators such as prostaglandin $E_{2}(P G E 2)$, leukotriene $E_{4}$ and lipoxin $A_{4}$ (LXA4) in mice plasma.

Methods: The effect of GLA pre-treatment on radiation induced inflammation was assessed by estimating plasma levels of high mobility group box 1 protein (HMGB1), TMOP/NO and various anti-oxidant enzymes.

Results: $\gamma$-linolenic acid pre-treated mice exposed to lethal IR dose (7.5 Gy) showed a decrease in plasma levels of HMGB1, PGE2 and LXA4 and a fall in TMOP/NO ratio and improvement in anti-oxidant enzymes: catalase, glutathione transferase and glutathione peroxidase compared to IR mice, suggesting that GLA suppresses IR-induced inflammation and restores the pro- vs. antioxidant ratio to near normal, which could explain its radioprotective action. Conclusions: GLA showed radioprotective action.

Key words: $\gamma$-linolenic acid, ionizing radiation, lipid mediators, inflammation, anti-oxidant enzymes.

Irradiation is known to induce the release of arachidonic acid (AA) and other polyunsaturated fatty acids (PUFAs) from the cell membrane lipid pool by activating phospholipase $A_{2}$ (PLA2) [1]. The released AA and other PUFAs (especially $\gamma$-linolenic acid (GLA), dihomo-GLA (DGLA), eicosapentaenoic acid (EPA) and docosahexaenoic acid (DHA)) can be converted to their respective metabolites such as pro-inflammatory prostaglandins (PGs), leukotrienes (LTs) and thromboxanes (TXs) and anti-inflammatory lipoxins, resolvins, protectins and maresins. Prostaglandins, LTs and TXs formed from AA are believed to be principally pro-inflammatory in nature whereas PGs, LTs and TXs derived from EPA are less pro-inflammatory (e.g. prostaglandin $E_{2}(P G E 2)>$ prostaglandin $E_{3}(P G E 3)$ ). Paradoxically, $A A$ can also form a precursor to potent anti-inflammatory lipoxin $A_{4}$ (LXA4), EPA to resolvins of E series and DHA to resolvins of D series, protectins and maresins. In addition, DGLA forms a precursor to anti-inflammatory prostaglandin $\mathrm{E}_{1}$ (PGE1) [2-7]. 
Previous studies revealed that GLA and its derivative PGE1 protect mouse hematopoietic stem cells and human lymphocytes from damage due to ionizing radiation and chemicals both in vitro and in vivo [8-14]. But the exact mechanism by which these cytoprotective and genoprotective actions are brought about is not known. Since PUFAs can form precursors to both pro- and anti-inflammatory metabolites, we hypothesized that treatment or supplementation of GLA and other PUFAs may enhance the formation of anti-inflammatory products that may be responsible for the radio-protective action shown by GLA in these studies. It is likely that protection of tissues from radiation-induced damage can occur only if there is an adequate inflammatory response to start with to trigger kick starting of the cytoprotective process [15-18]. Some of these molecules that are involved in the inflammatory process and subsequent resolution of inflammation, repair and restoration of homeostasis are bioactive lipids such as PGs, LTs, TXs, lipoxins, resolvins, protectins and maresin and pro- and antiinflammatory cytokines, lipid peroxides and free radicals. In order to understand their role in injury and recovery, it is important to measure their plasma and tissue concentrations at various stages of radiation-induced inflammation and resolution. Such an understanding of the fragile balance (homeostasis) between pro-inflammatory and pro-resolution molecules needs to be established post-irradiation so as to ensure the survival of the organism, which is indeed the main aim of developing successful radio-protective measures with minimal side-effects. The current study was designed to explore a timebased response of lipid mediators (both pro- and anti-inflammatory) against lethal ionizing radiation within the acute phase. The maintenance of homeostasis is also looked at by analyzing the ratios of various lipid mediators to gain an insight into the cyclo-oxygenase (COX) and lipoxygenase (LOX) mechanisms that are likely to be modulated by GLA. In the present study, we performed studies only with GLA since our previous studies revealed that of all the PUFAs tested GLA is the most effective lipid against radiation-induced damage [8-14].

Methods. $\gamma$-linolenic acid used in the study was procured from Cayman Chemical Company, Michigan, USA. The other necessary chemicals and reagents were procured from SRL Pvt. Ltd., India.

The current study was carried out using 8-10week old C57BL/6J female mice procured from the National Institute of Nutrition (Hyderabad, India). The animals were maintained at $25 \pm 2^{\circ} \mathrm{C}$ with a $12 \mathrm{~h}$ dark and $12 \mathrm{~h}$ light cycle. Animals weighing around 18-20 g were segregated into four groups containing 12 animals each for survival studies: controls treated with $0.02 \%$ ethanol in PBS, the GLA group receiving only GLA $100 \mu \mathrm{g} / \mathrm{kg}$ body weight as i.p. injections, the irradiation (IR) group treated with $0.02 \%$ ethanol in PBS and receiving $7.5 \mathrm{~Gy}$, and GLA + IR group animals pre-treated with GLA $(100 \mu \mathrm{g} / \mathrm{kg})$ at $48 \mathrm{~h}, 24 \mathrm{~h}$ and $1 \mathrm{~h}$ prior to receiving radiation. Animals were maintained with access to standard mice pellet feed (Nutrimix, Nurivet Life Sciences, Pune) and received water ad libitum. Separate sets of animals were segregated into four groups: control, GLA, IR and GLA + IR with 6 animals in each group for different time points including day 1 , day 3, day 7 and day 14 to study the antioxidant status and ELISA. The study was approved by the Institutional Animal Ethical Committee.

Animals (in cages restrained with fiber mesh) were subjected to total body irradiation with a lethal dose of 7.5 Gy (X-rays) using CLINAC IX (Varian Medical Systems India Pvt. Ltd.) at a dose rate $1 \mathrm{~Gy} / \mathrm{min}$.

Radio-protective efficacy of GLA $(100 \mu \mathrm{g} / \mathrm{kg})$ was studied upon pre-treatment with GLA at $48 \mathrm{~h}, 24 \mathrm{~h}$ and $1 \mathrm{~h}$ prior to exposure of animals to a lethal dose of $\mathrm{X}$-rays by performing 30-day survival assay. Death of animals was observed on a daily basis, twice a day. Survival was expressed as a percentage over a 30-day period in a Kaplan-Meier survival plot.

Control animals and those irradiated with or without GLA were euthanized at the end of different time periods day 1 , day 3 , day 7 and day 14 after radiation exposure. Blood was collected by cardiac puncture, plasma was separated from blood of mice collected in tubes coated with EDTA followed by centrifugation at $2500 \mathrm{~g}$ and stored at $-80^{\circ} \mathrm{C}$ until analyzed. The levels of high mobility group box 1 protein (HMGB1) (201205), PGE2 (514010) and leukotriene E4 (LTE4) (520411) were measured using ELISA kits from Cayman Chemical Company, Michigan, USA and lipoxin A4 (EA45, Oxford Biomedical Research Company, MI, USA) as per the manufacturer's instructions.

Plasma collected from animals (as mentioned above) was used for the estimation of antioxidant enzymes: catalase, superoxide dismutase, glutathione-S-transferase, glutathione peroxidase as described previously [19]. Lipid peroxides as malondialdehyde (MDA) formed on reaction with thiobarbituric acid (TBA) and nitric oxide (NO) measured as nitrite formed using Griess reagent were both estimated in the supernatant as described previously [19].

Experiments were performed at least twice in triplicate to ensure the repeatability of the results obtained. Data analysis was performed using the paired $t$-test using the MS Excel statistical analysis tool. All values are expressed as mean \pm SEM.

Results. Control and GLA treatment $(100 \mu \mathrm{g} / \mathrm{kg})$ did not induce any mortality in C57BL/6J female mice over the 30-day period observed. GLA + IR treated animals showed significantly increased survival (83.3\% against $16.7 \%$ ) compared to the IR group (Figure $1 \mathrm{~A}$ ). 
Treatment with GLA caused an initial significant increase in HMGB1 levels on days 1 and 3 and on days 7 and 14 the levels were still higher compared to control but were much lower compared to those seen on days 1 and 3. Irradiation caused a significant increase $(p<0.001)$ in HMGB1 levels at all time periods (days 1, 3, 7 and 14) post-irradiation compared to the control but the pattern was very similar to those treated with GLA alone (much larger increase on days 1 and 3 and much smaller increase on days 7 and 14). However, $\mathrm{GLA}+\mathrm{IR}$ animals exhibited significantly low levels of HMGB1 compared to IR at all time periods (Figure $1 \mathrm{~B}$ ) but the pattern again was very similar to that seen with GLA and irradiation treatments. These results suggest that irradiation produces a significant increase in plasma HMGB1 levels on all days examined with much higher spikes on days 1 and 3 that declined by days 7 and 14 but remained higher than the control values.

$\gamma$-linolenic acid treated animals exhibited low levels of PGE2 compared to control. However, irradiated animals showed significantly $(p<0.001)$ high levels of PGE2 compared to control at all time periods. Conversely, GLA + IR animals had significantly $(p<0.001)$ low levels of PGE2 compared to IR at all time periods (Figure $1 \mathrm{C}$ ) but higher compared to the control.

$\gamma$-linolenic acid treated animals had significantly $(p<0.001)$ higher levels of LTE4 compared to the control at all time periods with the highest peaks on day 1 and day 14 with slightly lower plateau values on days 7 and 14 . Surprisingly, irradiated animals showed values that were almost equal to those seen in the control, whereas IR + GLA animals showed values that were almost equal to those seen in the GLA alone treated group on day 1 , much higher values on day 7 compared to all other groups, and again much lower values equivalent to those seen in the control group by day 14 . On day 14, IR animals exhibited significantly $(p<0.001)$ low levels of LTE4 compared with the control. $\gamma$-linolenic acid $+I R$ animals showed a significant increase in LTE4 levels compared to IR at all the time periods (Figure $1 \mathrm{D}$ ).

Animals treated with GLA, irradiation and GLA + irradiation showed consistently higher values on all days except that on day 7, in GLA treated animals the levels were similar to those seen in the control (Figure $1 \mathrm{E}$ ). $\gamma$-linolenic acid treated animals showed a significant $(p<0.001)$ increase in plasma LXA4 levels on day 1 and 14. lonizing radiation also caused a significant $(p<0.001)$ increase in plasma LXA4 levels at all time points. $\gamma$-linolenic acid $+I R$ animals had significantly $(p<0.001)$ low levels of LXA4 on days 3, 7 and 14 compared to IR (Figure $1 \mathrm{E}$ ).
Since under physiological conditions one would expect a delicate balance between pro- and antiinflammatory bioactive lipids, we next examined the ratio between LTE4 and LXA4, PGE2 + LTE4 vs. LXA4 and LTE4 : LXA4/PGE2.

$\gamma$-linolenic acid treated animals exhibited a significantly $(p<0.01)$ high $\mathrm{LTE}_{4} / \mathrm{LXA}_{4}$ ratio at all time points with much higher peaks seen on days 1 and 14 , whereas there was a significant $(p<0.01)$ fall in the ratio in $I R$ animals from day 1 to day 14 and on all these days the values were consistently lower compared to GLA and GLA + IR treated animals. $\gamma$-linolenic acid $+I R$ animals showed an increased LTE4/LXA4 ratio on day 1 and day 7 and subsequently returned to normal by day 14 (Figure $2 \mathrm{~A}$ ). These results suggest that irradiation suppresses the production of LTE4 whereas GLA alone and GLA + irradiation treatment produce a significant increase in LTE4/LXA4 ratio.

The GLA group showed an increased $(p<0.01)$ (PGE2 + LTE4)/LXA4 ratio on day 1 and day 14 compared to the control. Irradiated animals showed an increased $(p<0.01)($ PGE2 + LTE4)/LXA4 ratio on day 1 , day 3 and day 7 (day $7>$ day $3>$ day 1 ) compared to the control and eventually it was restored to normal levels on day 14. $\gamma$-linolenic acid + IR showed a similar trend to that of IR, but there was a significant $(p<0.01)$ decrease in (PGE2 + LTE4)/ LXA4 ratio on day 7 and day 14 compared with IR (Figure $2 \mathrm{~B}$ ).

$\gamma$-linolenic acid treated animals displayed a significant $(p<0.01)$ increase in (LTE4 : LXA4)/ PGE2 ratio at all time points compared to the control whereas IR animals displayed a significantly $(p<0.01)$ decreased (LTE4 : LXA4)/PGE2 ratio at all time points compared to the control with peak values on day 1 . It can be seen that LTE4 : LXA4/PGE2 ratio is almost running parallel between GLA and GLA + IR treated groups except that the values are much higher in the GLA treated group. $\gamma$-linolenic acid + IR animals exhibited an initial increase in (LTE4: LXA4)/PGE2 ratio on day 1 compared to the control and IR but eventually it was restored to control levels by day 14 (Figure 2 C).

$\gamma$-linolenic acid treatment did not lead to any significant changes in TMOP/NO ratio at any of the time points studied. It can be seen that IR animals displayed a significant $(p<0.01)$ increase in TMOP/NO ratio at all time points; whereas, a gradual yet significant $(p<0.01)$ decrease was observed in GLA + IR animals from day 1 to day 14 compared to IR and it was restored to normal on day 14. Activities of the anti-oxidant enzymes catalase, GST and GPx which showed a significant decrease in irradiated animals reverted to normal by day 14 in GLA + IR-treated animals (Table I). 
A
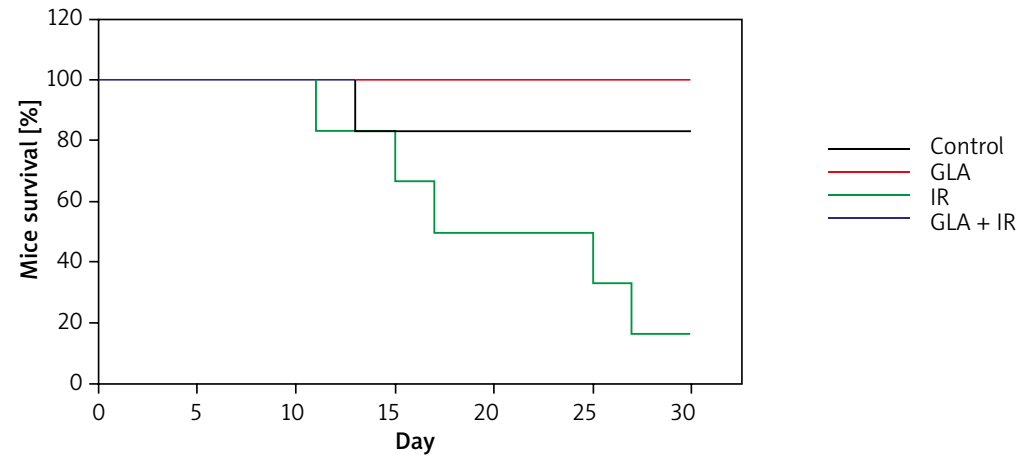

B

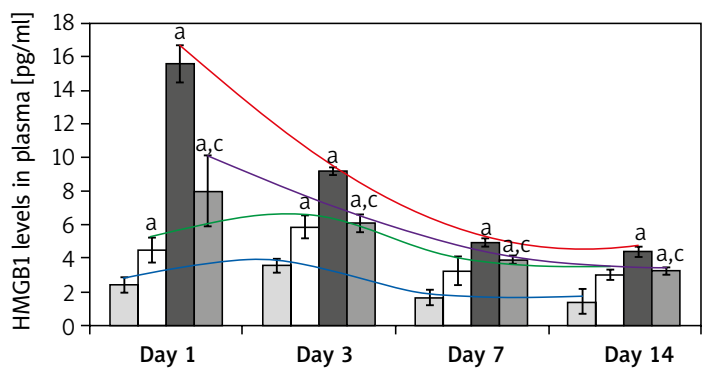

D

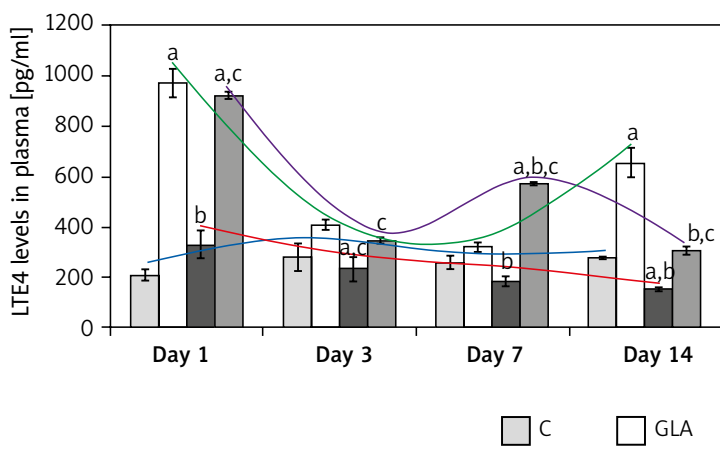

C

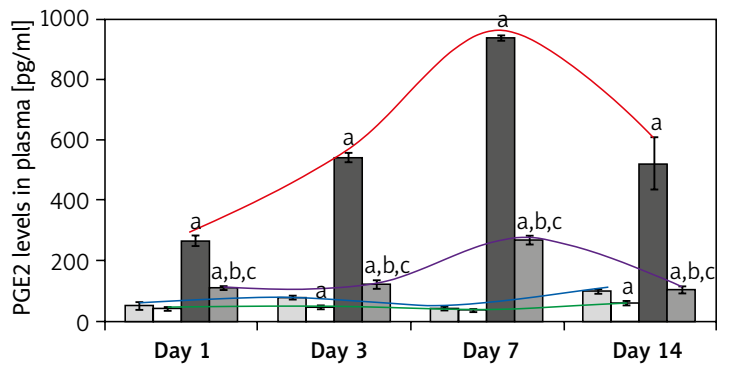

$\mathrm{E}$

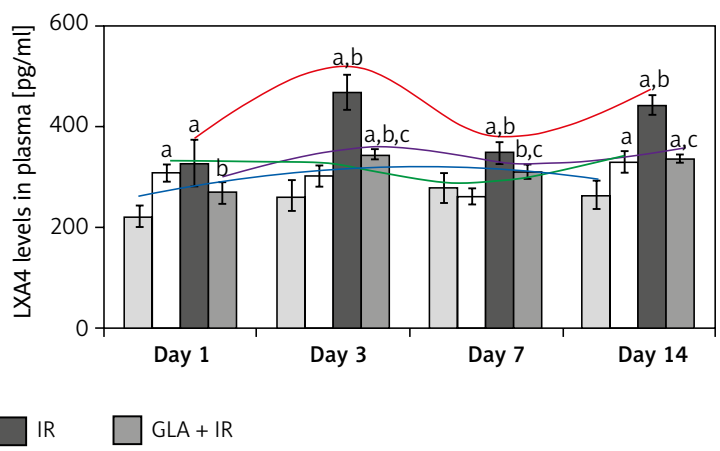

Figure 1. Survival in C57 BL/6J female mice. A - Mice pre-treated with $100 \mu \mathrm{g} / \mathrm{kg} \gamma$-linolenic acid (GLA) at 48, 24 and $1 \mathrm{~h}$ prior to irradiation and subjected to total body irradiation of $7.5 \mathrm{~Gy}$ (at $1 \mathrm{~Gy} / \mathrm{min})$. Values $(n=12)$ expressed as \% survival. B-E - Estimation of high mobility group box 1 protein (HMGB1) and polyunsaturated fatty acid (PUFA) metabolites by ELISA. Mice were pre-treated with $100 \mu \mathrm{g} / \mathrm{kg}$ GLA at 48, 24 and $1 \mathrm{~h}$ prior to irradiation and were subjected to total body irradiation of $7.5 \mathrm{~Gy}$ (at $1 \mathrm{~Gy} / \mathrm{min}$ ) and plasma was obtained on day 1 , day 3, day 7 and day 14 post-irradiation and estimated for (B) HMGB1, (C) prostaglandin $E_{2}$ (PGE2), (D) leukotriene $E_{4}$ (LTE4) and (E) lipoxin $\mathrm{A}_{4}$ (LXA4) by ELISA method. All the values are expressed as mean \pm SEM $(n=6)$. Statistical significance was calculated using $t$-test and $p$-value $(<0.001)$ is represented as ${ }^{\mathbf{a}, \mathbf{b}, \mathbf{c}}$ when compared to control, GLA and irradiation (IR) alone respectively

Discussion. The improved survival seen in $\mathrm{GLA}+\mathrm{IR}$ treatment compared to the IR group attests to the fact that GLA is protective against ionizing radiation (Figure $1 \mathrm{~A}$ ).

Irradiation is known to produce significant inflammation. This is supported by the observation that animals exposed to lethal irradiation (7.5 Gy) produced a significant increase in plasma HMGB1 and PGE2 levels on all days studied in the present study. Both HMGB1 and PGE2 are known to be pro-inflammatory molecules. In contrast, plasma LTE4, which is also a known pro-inflammatory molecule, concentrations were consistently low compared to the control on all the days studied
(Figure $1 \mathrm{D}$ ). These results are interesting, since previous studies showed that LTE4 possesses radioprotective action though it is much less effective compared to LTA4 and LTC4 [20-22] (Figure 3). Hence, it can be deduced from the results of the present study that the reduced levels of LTE4 may be responsible for the radiation-induced damage and mortality noted. The beneficial action of GLA in enhancing the survival of irradiation treated animals could be attributed to increased plasma levels of LTE4 noted in the GLA + irradiation treated animals since LTE4 has radioprotective actions. Since LTE4 is principally derived from AA whereas GLA is its precur- 
A

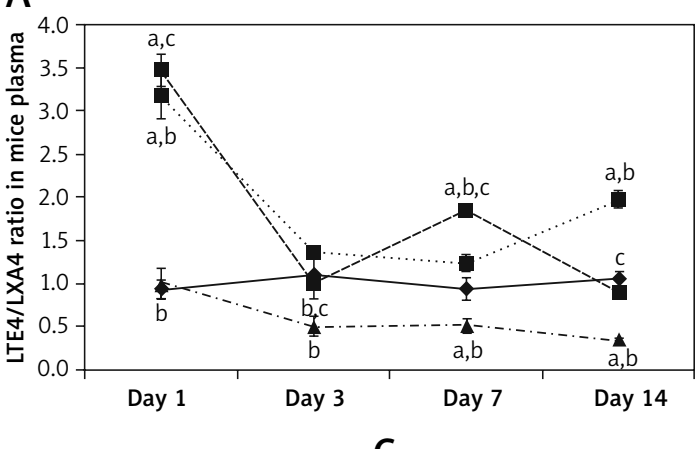

B

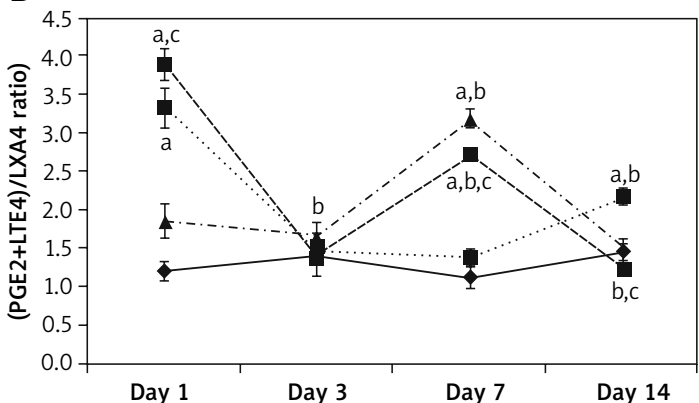

C

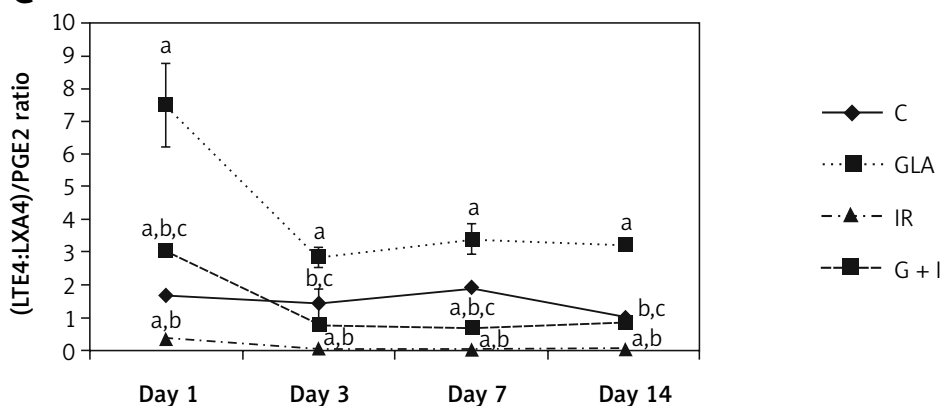

Figure 2. Ratio of polyunsaturated fatty acid (PUFA) metabolites in mice plasma: $A$ - leukotriene $E_{4}$ (LTE4)/lipoxin $A_{4}$ (LXA4), B - (prostaglandin $E_{2}$ (PGE2) + LTE4)/LXA4 and C - (LTE4 : LXA4)/PGE2. All the values are expressed as mean $\pm \operatorname{SEM}(n=6)$. Statistical significance was calculated using the $t$-test and $p$-value $(<0.01)$ is represented as ${ }^{a, b, c}$ compared to control, $\gamma$-linolenic acid and irradiation alone respectively

$C$ - control, GLA - $\gamma$-linolenic acid, IR - irradiation.

sor, it stands to reason to suggest that the administered GLA is elongated and desaturated to form AA that, in turn, is utilized to form increased amounts of LTE4 (from LTA4 that is initially formed from AA; Figure 3).

In this context, it is noteworthy that PGE2, which is also known to be a pro-inflammatory molecule derived from AA, has also been shown to possess radioprotective actions [23-28]. In the present study, it was observed that plasma PGE2 concentrations were elevated significantly from day 1 to day 14 in the irradiated group and GLA + irradiation groups compared to the control (irradiation group > GLA + irradiation; Figure $1 \mathrm{C}$ ). These results suggest that elevated plasma PGE2 levels may be a protective mechanism against radiation induced damage. Elevation in the plasma levels of PGE2 induced by GLA administration may be responsible for the increase in the survival of radiation-exposed animals (Figure $1 \mathrm{~A}$ ). Thus, changes in LTE4 and PGE2 levels induced by GLA treatment may be responsible for the beneficial action of GLA seen in the present study. It is interesting that pro-inflammatory molecules LTE4 and PGE2 are elevated in response to radiation and following GLA treatments and are responsible for the radioprotective action of GLA. This apparent paradoxical action can be attributed to the possibility that under some specific conditions PGE2 may serve as an anti-inflammatory compound and enhance the repair process and tissue regeneration [29-31]. It has been shown that blocking 15-PGDH (15-prostaglandin dehydrogenase) enzyme increases the half-life of PGE2 that results in enhancement in tissue regeneration and repair in the bone marrow, colon, and liver [31]. These results imply that perhaps an increase in the plasma levels of PGE2 due to radiation and GLA administration could be an attempt on the part of the body to augment hepatic, bone marrow and colon regeneration/repair, tissues that are commonly affected during radiation exposure and suppress inflammation. This is supported by the observation that HMGB1, a potent pro-inflammatory molecule, which showed significant elevation in its plasma levels of day 1 , showed a persistent and consistent decrease in its concentration on days 3, 7, and 14 . This suggests that despite an elevation in the levels of PGE2 and LTE4, which are considered as pro-inflammatory molecules, they may, in fact, function as antiinflammatory molecules under some very specific conditions, and hence a gradual and persistent decrease in HMGB1 levels was noted in the present study (Figure $1 \mathrm{~B}$ ). Thus, the elevations in the plasma levels of LTE4 and PGE2 noted in the present study in response to radiation and GLA treatments could be considered as radioprotective measures on the part of the body. 
Table I. Estimation of antioxidant enzymes, lipid peroxides and NO in C57BL6/J female mice plasma. Values ( $n=6)$ expressed as mean \pm SEM. Significance was calculated using paired $t$-test. ${ }^{a, b, c} p<0.01$ when compared with control, $\gamma$-linolenic acid and irradiation respectively

\begin{tabular}{|c|c|c|c|c|c|c|}
\hline Group & & $\begin{array}{l}\text { TMOP/NO } \\
\text { ratio }\end{array}$ & $\begin{array}{l}\text { SOD [units/ } \\
\text { mg protein] }\end{array}$ & $\begin{array}{c}\text { Catalase }\left[\mu \mathrm{M} \mathrm{H}_{2} \mathrm{O}_{2} /\right. \\
\text { min/g protein] }\end{array}$ & $\begin{array}{l}\mathrm{GPX}[\mu \mathrm{M} / \mathrm{min} / \\
\mathrm{g} \text { protein] }\end{array}$ & $\begin{array}{l}\text { GST }[\mu \mathrm{M} / \mathrm{min} / \\
\text { g protein] }\end{array}$ \\
\hline \multirow[t]{4}{*}{ Day 1} & Control & $0.38 \pm 0.01$ & $8.46 \pm 0.11$ & $45.51 \pm 6.78$ & $440.39 \pm 6.00$ & $0.69 \pm 0.05$ \\
\hline & GLA & $0.42 \pm 0.02$ & $9.44 \pm 0.11^{a}$ & $36.74 \pm 5.88$ & $463.21 \pm 3.20^{a}$ & $0.17 \pm 0.03^{a}$ \\
\hline & IR & $0.88 \pm 0.02^{\mathrm{a}, \mathrm{b}}$ & $7.07 \pm 0.32^{a, b}$ & $53.78 \pm 12.09$ & $486.12 \pm 5.16^{a, b}$ & $0.29 \pm 0.03^{a, b}$ \\
\hline & $G L A+I R$ & $0.71 \pm 0.02^{a, b, c}$ & $3.75 \pm 0.54^{\mathrm{a}, \mathrm{b}, \mathrm{c}}$ & $123.00 \pm 15.02^{\mathrm{a}, \mathrm{b}, \mathrm{c}}$ & $324.50 \pm 10.31^{a, b, c}$ & $0.50 \pm 0.04^{\mathrm{a}, \mathrm{b}, \mathrm{c}}$ \\
\hline \multirow[t]{4}{*}{ Day 3} & Control & $0.48 \pm 0.03$ & $10.36 \pm 0.27$ & $139.90 \pm 5.69$ & $5045.54 \pm 1072.74$ & $0.27 \pm 0.02$ \\
\hline & GLA & $0.48 \pm 0.02$ & $10.93 \pm 0.39$ & $150.61 \pm 6.14$ & $2300.51 \pm 81.97^{a}$ & $0.71 \pm 0.02^{a}$ \\
\hline & IR & $0.88 \pm 0.01^{\mathrm{a}, \mathrm{b}}$ & $10.15 \pm 0.59$ & $52.99 \pm 0.74^{a, b}$ & $2582.70 \pm 312.40^{a}$ & $0.32 \pm 0.03^{b}$ \\
\hline & $G L A+I R$ & $0.77 \pm 0.02^{a, b}$ & $14.23 \pm 0.54^{\mathrm{a}, \mathrm{b}, \mathrm{c}}$ & $115.43 \pm 18.82^{c}$ & $2848.60 \pm 118.88^{c}$ & $0.55 \pm 0.03^{b, c}$ \\
\hline \multirow[t]{4}{*}{ Day 7} & Control & $0.41 \pm 0.01$ & $4.96 \pm 0.25$ & $172.95 \pm 5.23$ & $2041 \pm 231.70$ & $0.14 \pm 0.02$ \\
\hline & GLA & $0.34 \pm 0.03$ & $15.28 \pm 0.31^{a, b}$ & $128.77 \pm 8.35^{a}$ & $2406 \pm 54.73$ & $0.28 \pm 0.03^{a}$ \\
\hline & $\mathrm{IR}$ & $0.77 \pm 0.03^{a, b}$ & $11.63 \pm 0.73^{b}$ & $321.89 \pm 13.33^{a, b}$ & $3134 \pm 200.36^{a, b}$ & $0.58 \pm 0.09^{a, b}$ \\
\hline & $G L A+I R$ & $0.52 \pm 0.01^{b, c}$ & $9.58 \pm 0.47^{a, b, c}$ & $205.51 \pm 18.36^{a, b, c}$ & $3458.99 \pm 126.06^{c}$ & $0.62 \pm 0.03^{a, b}$ \\
\hline \multirow[t]{4}{*}{ Day 14} & Control & $0.14 \pm 0.01$ & $22.25 \pm 1.98$ & $259.99 \pm 17.48$ & $142.36 \pm 16.95$ & $2.96 \pm 0.11$ \\
\hline & GLA & $0.22 \pm 0.04$ & $5.7 \pm 0.26^{a}$ & $226.76 \pm 31.8$ & $89.26 \pm 3.71^{a}$ & $1.38 \pm 0.08^{a}$ \\
\hline & $\mathrm{IR}$ & $0.32 \pm 0.004^{a}$ & $28.93 \pm 0.94^{a, b}$ & $118.48 \pm 22.37^{\mathrm{a}, \mathrm{b}}$ & $44 \pm 6.09^{a, b}$ & $1.06 \pm 0.09^{\mathrm{a}, \mathrm{b}}$ \\
\hline & $G L A+I R$ & $0.19 \pm 0.01^{c}$ & $17.83 \pm 0.73^{\mathrm{a}, \mathrm{b}, \mathrm{c}}$ & $344.58 \pm 55.02^{a, b, c}$ & $148.41 \pm 16.71^{\mathrm{b}, \mathrm{c}}$ & $2.09 \pm 0.18^{a, b, c}$ \\
\hline
\end{tabular}

GLA - $\gamma$-linolenic acid, IR - irradiation.

Arachidonic acid also forms a precursor to the potent anti-inflammatory metabolite LXA4. Since protection of tissues from radiation-induced damage needs anti-inflammatory events, we investigated whether there are significant alterations in the production of LXA4 in the present study in response to radiation and GLA treatment. As shown in the Figure $1 \mathrm{E}$, it is evident that plasma LXA4 levels were persistently elevated in GLA alone, radiation and GLA + radiation treated animals. Lipoxin A4 levels were very similar at all time points (within the groups) measured in GLA, GLA + radiation and control groups though the levels were significantly different among the groups (Figure $1 \mathrm{E}$ ). It is noteworthy that GLA alone treatment produced a significant increase in the plasma levels of LXA4 compared to the control and other groups with the highest peaks on day 3 and day 14 (day $3>$ day 14), while a significant dip in their levels was seen on day 1 and day 7 compared to the increases seen on days 3 and 14. It is noteworthy that the plasma levels of LXA4 were almost the same on day 1 and day 7 in the GLA alone treated group yet are significantly elevated compared to the control and other groups. On close examination of the data, it is evident that there is a reciprocal relationship among plasma LTE4, PGE2 and LXA4 levels (Figures $1 \mathrm{C}-\mathrm{E}$ ). It is noteworthy that whenever LTE4 levels are elevated there is a decrease in PGE2 levels and vice versa (Figures 1 C, D). For instance, on day 1 there was a significant increase in plasma LTE4 levels $\sim 1000 \mathrm{pg} / \mathrm{ml}$ whereas plasma PGE2 levels $\sim 300 \mathrm{pg} / \mathrm{ml}$ were comparatively low; on day 3, LTE4 levels were low but PGE2 levels were high; on day 7, LTE4 level were elevated to $600 \mathrm{pg} / \mathrm{ml}$ but were still much lower compared to PGE2 levels of $1000 \mathrm{pg} / \mathrm{ml}$; and on day 14, LTE4 levels were $\sim 200 \mathrm{pg} / \mathrm{ml}$ compared to PGE2 500 pg/ $\mathrm{ml}$. In a similar fashion, there is a inverse relationship in the plasma levels of LTE4 and LXA4 and a similar relationship is seen between plasma PGE2 and LXA4 levels (Figures 1 C-E). This suggests that there is a sort of a cyclical and inverse relationship among plasma LTE4, PGE2 and LXA4 levels. Since PGE2, LTE4 and LXA4 are all derived from the same precursor $A A$, this is an interesting observation implying that AA metabolism to form its pro- and anti-inflammatory metabolites is closely, tightly and finely tuned based on the necessity. Based on these results, it can be argued that there needs to be adequate inflammation to trigger the resolution process and this calls for an inverse and cyclical relationship observed in the plasma levels of PGE2/LTE4/LXA4 in the present study. Since GLA is able to mimic this natural process of an inverse and cyclical relationship among PGE2/LTE4/LXA4 

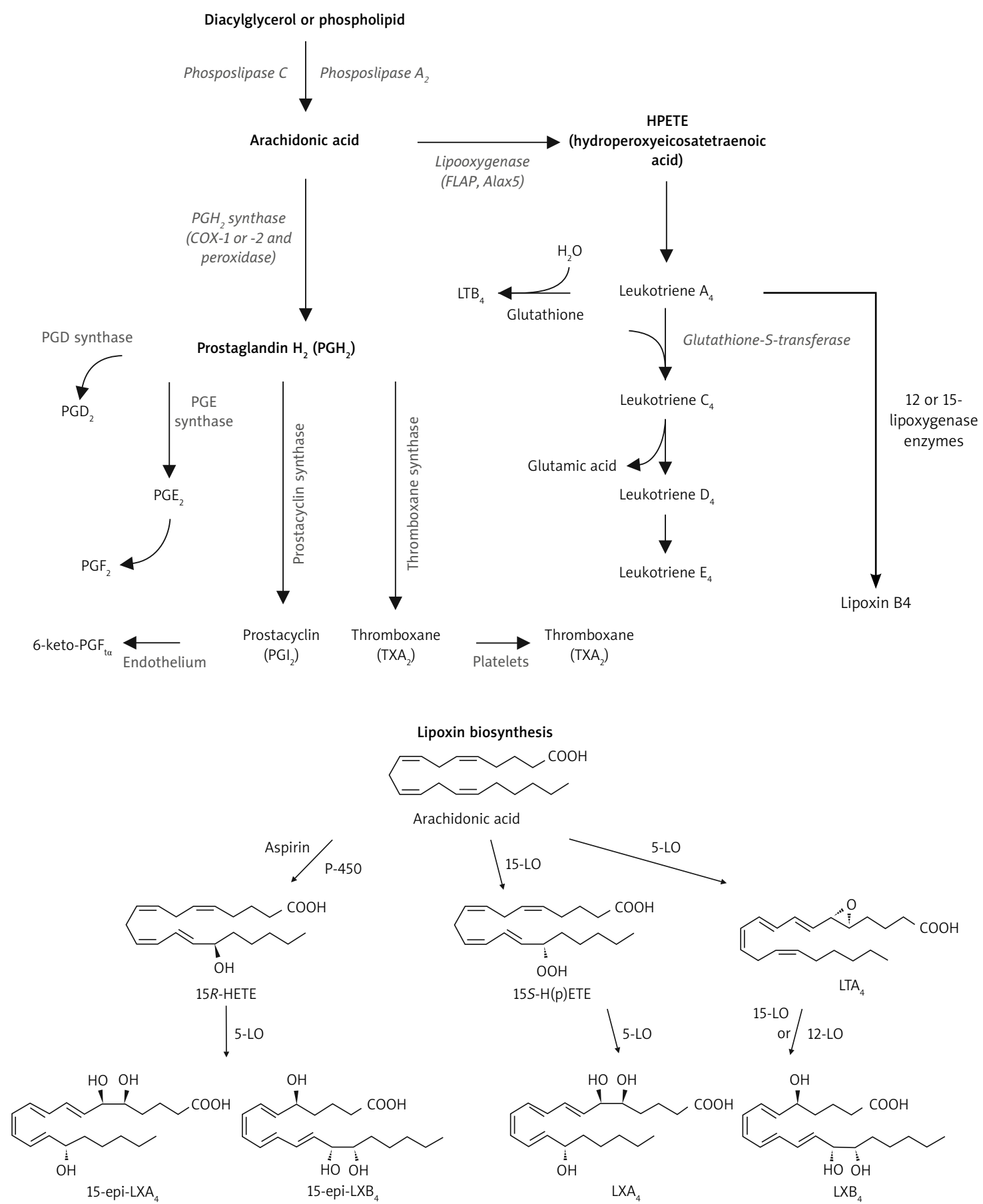

Figure 3. Scheme showing metabolism of arachidonic acid and various metabolites formed from it

levels, it is able to improve the survival of lethally irradiated animals in the present study. This is well brought out in Figure 2 wherein we calculated the ratios between LTE4/LXA4 (Figure $2 \mathrm{~A}$ ); PGE2 + LTE4/LXA4 (Figure 2 B) and LTE4-LXA4/ PGE2 (Figure $2 \mathrm{C}$ ). These calculations show that on day 1 there is an increase in pro-inflammatory molecules that were significantly decreased by day 3 ; an increase in their ratios occurred again on day 7 and fell by day 14 to the same level seen on day 3 . These results suggest that there is a need for an optimal inflammatory process to set in so that resolution of inflammation can be triggered. Since persistently elevated plasma/tissue PGE2 levels are needed to potentiate tissue regeneration, this may explain the elevated plasma PGE2 levels observed on all days of the study (Figure $1 \mathrm{C}$ ). Our recent study showed that administration of LXA4, a potent anti-inflammatory metabolite of $A A$, failed to show any radio-protection (unpublished data), once again emphasizing the fact that an optimal amount of initial inflammation is 
needed to trigger resolution of inflammation and tissue regeneration. In view of this, it is suggested that perhaps cyclical and alternate administration of pro- and anti-inflammatory molecules is needed to protect against radiation and other lethal tissue injuries as seen in sepsis.

\section{Acknowledgments}

UND conceived the idea, planned the studies and provided the reagents. This study was performed at BSRC under the guidance of UND. R.P is a Senior Research Fellow of University Grants Commission (UGC), New Delhi, India and the work presented in this paper is part of her Ph.D. work. This work was supported by a grant from Defence Research and Development Organisation (DRDO), New Delhi ((TC/2519/INM-03/2011/CARS) under R\&D Project INM-311) to UND. We acknowledge Mr. V. R. Senthil (SBC, DRDO) and Mr. Ananda Krishnan (Queen's NRI Hospital) for carrying out the animal irradiation procedures.

\section{Conflict of interest}

The authors declare no conflict of interest.

\section{References}

1. Gresham A, Masferrer J, Chen X, Leal-Khouri S, Pentland AP. Increased synthesis of high-molecular-weight CPLA2 mediates early UV-induced PGE2 in human skin. Am J Physiol 1996; 270: C1037-50.

2. Poorani R, Bhatt AN, Dwarakanath BS, Das UN. COX-2, aspirin and metabolism of arachidonic, eicosapentaenoic and docosahexaenoic acids and their physiological and clinical significance. Eur J Pharmacol 2016; 785: 116-32.

3. Das UN, Bhagat U. Potential role of dietary lipids in the prophylaxis of some clinical conditions. Arch Med Sci 2015; 11: 807-18.

4. Das UN. Lipoxins, resolvins, and protectins in the prevention and treatment of diabetic macular edema and retinopathy. Nutrition 2013; 29: 1-7.

5. Das UN. Radiation resistance, invasiveness and metastasis are inflammatory events that could be suppressed by lipoxin A (4). Prostaglandins Leukot Essent Fatty Acids 2012; 86: 3-11.

6. Serhan CN, Chiang N, Dalli J, Levy BD. Lipid mediators in the resolution of inflammation. Cold Spring Harb Perspect Biol 2014; 7: a016311.

7. Serhan CN. Discovery of specialized pro-resolving mediators marks the dawn of resolution physiology and pharmacology. Mol Aspects Med 2017; 58: 1-11.

8. Das UN, Rao KP. Effect of gamma-linolenic acid and prostaglandins E1 on gamma-radiation and chemical-induced genetic damage to the bone marrow cells of mice. Prostaglandins Leukot Essent Fatty Acids 2006; 74: 165-73.

9. Devi GR, Das UN, Rao KP, Rao MS. Prevention of radiation-induced poly-chromatophilia by prostaglandin E1 and colchicine. IRCS Med Sci 1983; 11: 863-4.

10. Das UN, Devi GR, Rao KP, Rao MS. Modification of benzo(a) pyrene induced genetic damage to the bone marrow cells of mice by prostaglandins. IRCS Med Sci 1983; 11: 823-4.
11. Ramadevi G, Das UN, Rao KP, Rao MS. Prostaglandins and mutagenesis: Prevention and/or reversibility of genetic damage induced by benzo(a)pyrene in the bone marrow cells of mice by prostaglandins E1. Prostaglandins Leukot Med 1984; 15: 287-91.

12. Das UN, Ramadevi G, Rao KP, Rao MS. Prostaglandins can modify gamma-radiation and chemical induced cytotoxicity and genetic damage in vitro and in vivo. Prostaglandins 1989; 38: 689-716.

13. Ramadevi G, Das UN, Rao KP, Rao MS. Prostaglandins and mutagenesis: modification of phenytoin induced genetic damage by prostaglandins in lymphocyte cultures. Prostaglandins Leukot Med 1984; 15: 109-13.

14. Das UN, Ramadevi G, Rao KP, Rao MS. Prostaglandins and their precursors can modify genetic damage-induced by gamma-radiation and benzo(a)pyrene. Prostaglandins 1985; 29: 911-20.

15. Das UN. Current and emerging strategies for the treatment and management of systemic lupus erythematosus based on molecular signatures of acute and chronic inflammation. J Inflammation Res 2010: 3: 143-70.

16. Fadok VA, Bratton DL, Konowal A, Freed PW, Westcott JY, Henson PM. Macrophages that have ingested apoptotic cells in vitro inhibit proinflammatory cytokine production through autocrine/paracrine mechanisms involving TGF-beta, PGE2, and PAF. J Clin Invest 1998; 101: 890-8.

17. Mitchell S, Thomas G, Harvey K, et al. Lipoxins, aspirintriggered epi-lipoxins, lipoxin stable analogues, and the resolution of inflammation: stimulation of macrophage phagocytosis of apoptotic neutrophils in vivo. J Am Soc Nephrol 2002; 13: 2497-507.

18. Byun JY, Youn YS, Lee YJ, Choi YH, Woo SY, Kang JL. Interaction of apoptotic cells with macrophages upregulates COX-2/PGE2 and HGF expression via a positive feedback loop. Mediators Inflamm 2014; 2014: 463524.

19. Polavarapu S, Mani AM, Gundala NK, Hari AD, Bathina S, Das UN. Effect of polyunsaturated fatty acids and their metabolites on bleomycin-induced cytotoxic action on human neuroblastoma cells in vitro. PLoS One 2014; 9: e114766.

20. Walden Jr TL, Patchen ML, MacVittie TJ. Leukotriene-induced radioprotection of hematopoietic stem cells in mice. Radiat Res 1988; 113: 388-95.

21. Walden Jr TL. Radioprotection of mouse hematopoietic stem cells by leukotriene A4 and lipoxin B4. J Radiat Res 1988; 29: 255-60.

22. Walden TL Jr. Leukotriene C4-induced radioprotection: the role of hypoxia. Radiat Res 1992; 132: 359-67.

23. Hanson WR, DeLaurentiis K. Comparison of in vivo murine intestinal radiation protection by E-prostaglandins. Prostaglandins 1987; 33: 93-104.

24. Geng L, Hanson WR, Malkinson FD. Topical or systemic 16, $16 \mathrm{dm}$ prostaglandin E2 or WR-2721 (WR-1065) protects mice from alopecia after fractionated irradiation. Int J Radiat Biol 1992; 61: 533-7.

25. Geng L, Hanson WR, Malkinson FD. Topical or systemic $16,16 \mathrm{dm}$ prostaglandin E2 or WR-2721 (WR-1065) protects mice from alopecia after fractionated irradiation. Int J Radiat Biol 1992; 61: 533-7.

26. Malkinson FD, Geng L, Hanson WR. Prostaglandins protect against murine hair injury produced by ionizing radiation or doxorubicin. J Invest Dermatol 1993; 101 (1 Suppl): 135S-7S.

27. Riehl T, Cohn S, Tessner T, Schloemann S, Stenson WF. Lipopolysaccharide is radioprotective in the mouse intestine through a prostaglandin-mediated mechanism. Gastroenterology 2000; 118: 1106-16. 
28. Riehl TE, Foster L, Stenson WF. Hyaluronic acid is radioprotective in the intestine through a TLR4 and COX-2mediated mechanism. Am J Physiol Gastrointest Liver Physiol 2012; 302: G309-G16.

29. FitzGerald GA. Bringing PGE2 in from the cold. Science 2015; 348: 1208-9.

30. Duffin R, O'Connor RA, Crittenden S, et al. Prostaglandin E2 constrains systemic inflammation through an innate lymphoid cell-IL-22 axis. Science 2016; 351: 1333-8.

31. Zhang Y, Desai A, Yang SY, et al. Inhibition of the prostaglandin-degrading enzyme 15-PGDH potentiates tissue regeneration. Science 2015; 348: aaa2340. 